\title{
Issues and Problems in the Blending of Cognitive Science, Evolutionary Psychology, and Literary Study
}

\author{
Tony E. Jackson \\ English, North Carolina at Charlotte
}

The present issue of Poetics Today brings together seven different writings that in one way or another have to do with the interdisciplinary mix of cognitive science, evolutionary psychology, and literary studies. For the sake of discussion I will separate the essays into different kinds based on different qualities, even though all share the qualities to some degree. We have samples of two main kinds of interdisciplinary work, namely metalevel discussions of large issues and working examples of practical interpretation. In the first category we begin with Mark Turner, who gives us a generalized overview of the current situation and its prospects and focuses on one interpretive device, conceptual blending, which he considers to be the primary crossover concept from the realm of cognitive studies into the realm of the humanities. We get from Ellen Spolsky a different overview of the issues. In terms of both evolutionary theory and cognitive psychology, she shows why no absolute divide need exist between empirically based critical approaches and the post-structuralist approaches that still dominate literary studies. From Paul Hernadi we get what might be considered a ground laying of fairly general ideas about "literature's role in the coevolution of human nature and cultures." Reuven Tsur, extrapolating from studies of prosody, makes the case that cultural forms or programs "have solid cognitive foundations and are shaped and constrained by the natural capacities and constraints of the human brain." The remaining essays, though they all make at least some generalized claims, are most notable for giving us

Poetics Today 23:1 (Spring 2002). Copyright @ 2002 by the Porter Institute for Poetics and Semiotics. 
models of the other main kind of interdisciplinarity, practical interpretation. Within this group we have examples of the two primary kinds of practical criticism that we are most likely to see. Lisa Zunshine and Francis Steen use concepts from cognitive scientific studies to interpret specific literary texts. Alan Richardson uses what cognitive science now shows us about the mind to look anew at the way older versions of mind-both scientific and folk-psychological-operated in literary texts. Of course other applications are possible, but bringing specific concepts to bear in specific interpretations (as Zunshine and Steen do) and examining historical versions of mind/body to interpret specific texts (as Richardson does) give us the two broadest categories of application.

As respondent I look at these essays as examples of an emerging interdisciplinary field. In my discussion I try to illuminate certain general issues that in my view ought to matter to anyone trying to bring cognitive science, evolutionary psychology, and humanistic studies together. For this reason I have created subtitled sections that address certain of these general concerns. First, we consider what we may call interdisciplinary necessity: if we are to have an inter-disciplinarity, then we require some reasonable necessity for bringing the concepts or methods of one discipline into working relation with the concepts or methods of another discipline. Our focus in this respect will be conceptual blending. Second, we consider the issue of the different kinds of truth production involved. Next, we look at how current literary theory and practice can be compared to certain key concepts in cognitive science and evolutionary psychology. Then we discuss two recurring issues in evolutionary psychological explanations of culture: the specter of social Darwinism and the speculativeness of claims. In the fifth section we investigate how arguments based on empirical science of whatever kind can be expected to hold a reasonable significance for a humanities audience. Sixth, we have a look at interdisciplinary necessity from the angle of what we may call the historical study of the mind/brain in literature. Next, we consider some likely extensions of some of the ideas included in this volume. Finally, we consider what I take to be one of the primary difficulties of any blend of an empirical scientific and a humanistic discipline.

In a sense I try to make plain the kinds of objections hovering in the background of what has actually been written. As we proceed we need to remember that these are early entries in cognitive literary studies. These writers are still working out fundamental concepts and operating procedures. In fact in the future we may well look back at these kinds of attempts as part of a phase or stage on the way to a more full-blown interdisciplinarity to come. My response hopes to help push things along. 


\section{Conceptual Blending and the Issue of Interdisciplinary Necessity}

Mark Turner is wise to begin his general explanation of the cognitive study of art, language, and literature by situating the new theory within the history of classical rhetoric. This is wise because he thereby plants cognitivescientific study of the humanities in a historical field that students of the humanities will recognize and acknowledge and he avoids the evangelical tone that sometimes shows up in writings proclaiming a revolution in criticism. But on the other hand he takes a necessary risk in doing this because he will now have to show how his new approach to the humanities significantly differs from classical as well as contemporary rhetoric. If he fails to make the case for a real and critically productive difference between the new and the already established approaches, the cognitive sciences simply may not become much of a force in the humanities. So when he speaks of "conceptual blending," for instance, which is thus far one of his own mostresearched ideas and one of the main imports from cognitive studies to literary studies, we may ask: Will this idea be sufficiently different from discussions of metaphor (to take the most likely figure) that might be possible without bringing in another disciplinary perspective? Do we really need cognitive science to do what Turner describes?

The answer is yes and no. On the one hand he provides us with a strikingly useful method for unpacking figurative language, be it in literary or everyday use. We linguistic creatures operate constantly, without having to think about it, by "combining two mental packets of meaning - two schematic frames of knowledge or two scenarios, for example-selectively and under constraints, to create a third mental packet of meaning that has new, emergent meaning." To use some of his other terms, we map selected elements from one mental space onto elements of another space, and the result is a third space whose properties are more than any sum of the two. This "advanced ability for conceptual integration" is, he argues, "the central capacity of cognitively modern human beings." Turner gives us a number of analyses of specific blends to help make his case: "This surgeon is a lumberjack," for example. We have one packet of meaning associated with surgeons, the other with lumberjacks, but the actual meaning we get is a third packet created, automatically in the majority of cases, from only part of the possible elements in each source packet. Other writings of his have provided yet more, equally potent examples (Turner 1996). In becoming aware of this operation in this way we gain what seems to me a valuable method of looking at figurative language of whatever kind. I would recommend an understanding of conceptual blending as an interpretive tool to any student of literature or rhetoric because it provides a systematic way 
of identifying qualities or elements that do and do not become involved in figurative speech. The basic idea of conceptual blending then is quite useful. Also it seems indisputably true to the facts of language.

But then, to get to the "no" side of my "yes and no," it must also be said that the idea is equally indisputable without cognitive science to make the case. As far as I can tell, if we set out to unpack any metaphor or other linguistic figure and we consider the relevant elements of the (at a minimum) two items brought together to render a third meaning, we can use much the same method Turner does without knowing anything about cognitive science. Turner himself shows an awareness of this objection when he acknowledges in a footnote that Arthur Koestler in The Act of Creation (1964) had already in a roundabout way made use of the concept of blending. ${ }^{.}$But Koestler regarded "blending as exceptional" rather than as a fundamental activity of the everyday mind, and he had "no theory of its structural and dynamic operation." True enough, but this did not prevent Koestler from actually doing more or less the same kind of interpretation Turner does. Another hint that Turner is trying to head off this objection is found in his abstract. There he writes that the "cognitive turn in the humanities . . . draws much of its content, many of its central research questions, and many of its methods from traditions of the humanities as old as classical rhetoric." What Turner does not say is that his particular interpretive methodology may have been generated and perfected by his studies in cognitive sciences, but the methodology works quite well without the science as simply another, smart version of ancient methods of analyzing figurative language. ${ }^{2}$

\section{The Issue of Epistemologies}

We are led to ask: How can the cognitive sciences (we will consider evolutionary psychology below) matter for the humanities? The essays of practical interpretation will help answer these questions, but let us first look generally at the present state of interactions between literary (for it is with this one of the humanities that I deal) and cognitive scientific study. At this dawning interdisciplinary moment, we commonly find some form of the following epistemological process. In one or more of the current areas of cognitive-scientific study-cognitive psychology, cognitive anthropology, cognitive rhetoric, cognitive linguistics - certain facts about human cogni-

1. Turner (1996: 72) also mentions Koestler in The Literary Mind.

2. The same might possibly be said of Turner's claims about cognitive neuroscience in relation to history. But he is giving us only a general introduction in this respect. Given the context in which the article was delivered, he simply lacked time to give us an example, so it is not easy to see how exactly this mix would actually work. 
tive architecture have been (at least) adequately established through empirical research. Sometimes these facts have been augmented by neurological research on the brain as the biological cause of the mind. Along with this we often find an analogical process at work in which generalizations are made at a level of abstraction sufficient to enable us to compare concepts across the three areas of knowledge, that is, neuroscience, cognitive science, and literary study. In what seems to be the ideal model, the brain is shown by neuroscience to perform a certain function in a certain way, the architecture of a certain mental capacity is shown by cognitive science to operate in a way analogous to the brain function, and a psychological or cultural artifact or process is shown to operate in the same analogous way.

In The Literary Mind, for instance, Turner (1996: 23-25) brings in neuroscientific claims about perceptual image schemata, which support psychological claims about conceptual image schemata, which in turn back up his claims about linguistic conceptual blends. He does much the same with his discussion of integration as an aspect of blending (109-15). In this manner a writer can make claims about literature that, at least compared to the usual literary argument, are scientifically based. The degree of scientificity does vary. Turner uses brain science sparingly and judiciously. "It is important," he writes in the essay included here, "to realize that having one foot in cognitive neuroscience does not mean uncritically adopting the ideas of cognitive neuroscientists for recycling within literary studies." But scientific empiricism matters, and perhaps unavoidably the support of neuroscience, the "hardest" science in all this, seems always to be expected (not just by Turner) as a final arbiter of the claims all along the line.

And why not? The urge for more solid forms of truth making in literary interpretation flowers up regularly, and scientific truth, even when we are hyperskeptical of it, retains a kind of argumentative solidity that has few equals. But literary interpretation as it is commonly practiced seems to me to lie somewhere in the uncomfortable middle of a continuum of truth production that runs from hard science on the one end to the "truths" we find in artworks on the other. I do not mean to say of course that scientists have no aesthetic element in their work or that artists are all natural geniuses, but still this distinction carries weight. As one result scientists (many at least) consider our literary-interpretive claims to be purely subjective if not goofy, and artists (many at least) consider our literary-interpretive claims to be murder by academic dissection. To the scientists we are would-be artists; to the artists, we are would-be scientists. And I for one have to say that at times I feel a certain agreement with both sides, which is what must be expected of being in the middle of this continuum. What I write interpretively about a given text can seem, even though it may be publishable, uncomfortably 
nebulous as a truth claim if I think of what I write in relation to the kinds of claims made in certain other disciplinary contexts. At the same time I can feel not quite right about the entire operation of recasting the text out of itself and into the form of discursive argument, just as you might do with any old object in the world that you wanted to explain. I take it that literature is not just any old object in the world but is in fact a special kind of linguistic object (though I can make the arguments to show that I am wrong about this specialness).

Those who bring cognitive scientific and evolutionary psychological studies to bear on literary interpretation also, as far as I can tell, take literature to be a special linguistic object, but not special in such a way that it would fall altogether outside scientific or science-based explanations. For them to consider literature and interpretation apart from what the cognitive sciences have to say about the "embodied mind" is in a way to do with literature what René Descartes gets credit for doing with the mind: separating it from the flesh and the blood and the foul rag and bone shop of the beating heart. But of course in blending William Butler Yeats with Descartes just now I have confused things. Yeats did not really mean the heart as a biological organ, and it is hard to imagine him having much time for anyone who would bring in the biological factuality of that organ in a discussion about its importance either to literature or to that part of the human being that can be seen as somehow other than biological. Yeats, and likely most poets, would agree that literature has much to do with humans in their animality but would see the literary investigation of that situation as entirely different from anything like a scientific investigation of it. He would have lots of company, I suspect, among literary scholars.

Of course many of those same scholars, I suspect, would likely have a pretty strong, if not absolute, commitment to the idea that a human being in principle is ultimately biological and only biological. If the mind is a function of the brain and body, as I assume most scholars would admit, then why should not biological explanations be relevant for whatever human activity we might consider? The answer of course is that the biological explanation simply may not be relevant to all levels of investigation. Though all we think and do may be the outcome of biological processes, some of these outcomes are too far removed from those ultimate causes, to an extent that biology just will not explain the actual subject of inquiry.

The next question is: Given this, how exactly are biological and, say, literary explanations related? At one extreme it may be that biological explanations are to psychological and cultural affairs as quantum mechanics is to the everyday Newtonian universe. At some point in specifying an explanation of, for instance, how a surgeon's knife moves through flesh, we 
would presumably come to a quantum level of matter. After all the flesh is made up of cells that are made up of molecules that are made up of atoms that are made up of subatomic (voilà!) particles. Yet though all this is true, quantum explanations are not generally relevant to everyday physics.

Such a comparison probably overstates the difference of levels between biology and culture. In fact cognitive science appeals in part, it seems to me, because it falls in between strictly biological explanations and the usual literary-interpretive explanations. Its claims arise from empirical studies but primarily from studies of people doing relatively people-y things. At least it investigates a mental organ. Therefore it can be seen as providing a possible anchor for interpretations, so truth production does not seem like little more than a function of disciplinary fashion. At the same time it can be seen as not constraining interpretation in a hard-science way.

Empiricism of whatever scientific variety cannot be brought into the discussion without certain consequences. At times these writers are subject to questions from the scientific side about their understanding of the science and their use of the science outside its immediate contexts. At other times they are equally subject to questions from the literary-critical side about the entire idea of explaining literature by means of any overtly scientific concept or approach. Whatever else may be said, it takes a blend of nerve and intellectual curiosity even to attempt to push into this interdisciplinary frontier.

\section{The Issue of Interdisciplinary Compatibility}

Opening up the frontier in a way that others may follow requires a sense of both sameness and difference. We have had a number of statements of difference, of how cognitive and evolutionary studies will revolutionize the study of literature by overthrowing the rule of poststructuralism (Turner 1991; Carroll 1995; Storey 1996). But here we also have Ellen Spolsky calmly and clearly explaining the conceptual similarities between certain key poststructuralist conclusions and certain key claims of contemporary Darwinism and cognitive psychology, all of which she understands quite well. Yes, this blend of disciplines will be critically different; no, it will not necessarily banish all the literary-interpretive concepts presently in operation. She brings together the findings of poststructuralism about the instability of meaning, Stanley Cavell's more optimistic understanding of the same idea, general concepts of evolutionary theory about the necessary imprecision of natural processes, and the modularity theory of cognition. In this case the primary analogy finds that contemporary Darwinism "is attractive to literary theory because the processes it hypothesizes for the natural world 
of plants and animals, that is, spontaneous change/variation followed by survival and loss and temporarily stable subspeciation, are consistent with many of the most interesting recent theories of mind, knowledge, meaning, and interpretation." She shows by analogy how writings by Noam Chomsky and Ludwig Wittgenstein about language, Stephen Greenblatt about the "circulation of social energy," and Lorraine Code about feminist epistemology all bear strong conceptual similarities to Darwinian theory. This is useful ground-laying work that obviously hopes to head off a certain poststructuralist skepticism at the pass.

\section{Interdisciplinary Issues with Evolutionary Psychology}

Most writers who bring these relatively scientific disciplines into contact with literary studies are strongly aware of at least one thorny, fundamental epistemological issue: the causal relationship between the realms of biology and the realms of culture and psychology. As I have argued in an earlier number of Poetics Today (Jackson 200o), getting things wrong with this issue can be especially serious because it can easily lead into not just any misconception of the actual nature of things but into a misunderstanding that can then lead straight into some version of social Darwinism. In part, social Darwinism succeeds as a putatively scientific notion because of the mistaken idea that biology simply causes psychology or culture. All of the writers here understand this situation, and all attempt to offset it, typically by making the case for a dialectical understanding of cause and effect.

We find this well illustrated in Paul Hernadi's hypothesis about the coevolution of literature and the brain. To put it simply, we may say that the most common notion of evolutionary change runs as follows: over a very long period of time a random genetic mutation happens to lead to some larger-scale, viable, new physiological and/or anatomical element, which, if the environment happens to be favorable, leads to a reproductive advantage, which leads to an increase in the number of creatures endowed with the new element. With respect to cause and effect, this scenario tends to move in one direction. The environment plays a crucial but essentially passive part. But when we come to evolutionary psychology, we will probably need a much more dialectical notion of cause and effect. For this reason such books as Terrence Deacon's The Symbolic Species (1997) have become an important source for evolutionary-psychological arguments. To oversimplify again, Deacon argues against the idea that the evolution of an anatomical change from a smaller to a larger brain brought about language and thus modern human beings in a more or less direct causal sequence. A larger brain was a necessary but not sufficient condition for the emergence 
of Homo sapiens. Rather, language itself played as large a role in determining the nature of the brain as vice versa. According to Deacon (1997: 45) the "human brain should reflect language in its architecture the way birds reflect the aerodynamics of flight in the shape and movements of their wings." If language coevolved with the brain and if literature is a specifiable, universal kind of language, then can a case be made for literature's part in evolutionary psychology? Hernadi says yes and offers us some specific hypotheses by which to consider this conceptual extension from the idea of the coevolutionary effects of language. Citing generally agreed-upon qualities of literature and literary experience, he explains how these qualities might have led to certain reproductive advantages in early human life. His essay is an exemplary model for this kind of argument, especially because he is so clearly aware of the generic difficulty with all such argumentsthey seem like just-so stories that cannot be proved in the way of, say, arguments for biological evolution. For this reason he judiciously qualifies his language, writing always that an element of literature could have or might have helped bring about a certain advantage. Still, just here is where interdisciplinarity becomes as much a problem as a solution. Hernadi is a fine writer, yet his essay suffers unavoidably from the use of such words as may and can at the most important moments.

For a close look at our main example of evolutionary psychology, consider the following statement by Hernadi: "I do not claim to know what kind of neurological evidence would corroborate or undermine the folk psychological intuition that human emotion, cognition, and volition are both distinguishable and intertwined dimensions of the mind." This statement rather perfectly captures some of the tensions built into evolutionary psychology as a discipline. We see the strong desire for a hard-science grounding in neurology, which may be difficult or in some sense impossible to obtain for these elements of human being. ${ }^{3}$ Along with this we see the compelling interest of folk psychology. Folk psychology is of course nonscientific, may be leaky with self-contradiction, and often enough is not even articulable by the "folk." Still it is a quite specifiable, real, and powerful understanding of the mind. On the one hand, because folk psychology in some form exists universally, it must have emerged over time as a result of regularly occurring interactions between biologically similar human beings in relatively stable environments; it must then somehow be based on ultimately biological predispositions. For this reason neuroscience might possibly "corroborate" folk psychology. On the other hand,

3. Yet cognitive science has already pushed into such categories. Perhaps the most famous example of this would be Damasio 1994 . 
because folk psychology is a cultural phenomenon, it may be simply untrue to the neurological facts and therefore undermined by neurology in much the way that racism is untrue to and therefore undermined by the genetic facts. In any event neuroscience or some other empirical science automatically takes place as the final judge of the cultural or psychological phenomenon. Until we have that kind of grounding, evolutionary-psychological arguments will seem relatively speculative, necessarily claiming only what "may" have been. In elaborating this dilemma, I suspect I say nothing that Hernadi himself would not acknowledge, nor do I want to discourage this kind of thinking. We are still early in our efforts to relate cultural and psychological affairs to contemporary evolutionary theory. Writings such as Hernadi's are setting methods and directions for further thought.

\section{The Issue of Interdisciplinary Significance}

Because Reuven Tsur's "Some Cognitive Foundations of 'Cultural Programs" is perhaps the most thoroughly mixed case of theory and application here, I turn to it as a transition from the more metalevel to the more directly interpretive essays. In some ways this piece is also exemplary. The first third of the essay makes the case for the ways certain cognitive universals can explain the historical appearance of certain more or less universal elements of prosody. This involves combining cognitive psychological claims about short-term memory, gestalt rules of perception, and what amount to statistical surveys of poetic lines with a view to formulating specific "intercultural principles." The next third of the essay explains the ways "in which intercultural principles have been individuated," which is to say, how differences among these universals can be accounted for, at least in general ways. The last third discusses some possible counterexamples to his claims.

Making the case for sameness (cognitive universals) and difference will of necessity be a central requirement for much work in this kind of interdisciplinary endeavor. Just claiming that a cognitive universal is operating may be true but will not usually be an act of literary interpretation. To be interpretive a writer will have to make some claim about what it means that a cognitive universal is operating in just the way it is in just this particular kind of textual example. Nevertheless Tsur's essay is also exemplary because, even though he deals with difference, the kind of difference explained is a kind that will find only a small audience among literary scholars. $\mathrm{He}$ himself says his main example of prosodic conventions "may appear marginal to many readers." Another way of saying this is that his explanation of difference, the individuation of the universals, does not really move out of the realm of science into the realm of culture. In the last paragraph of his 
essay Tsur alludes to just what I mean when he speaks of the metaphysical conceit as a form of "adaptation devices turned to aesthetic ends" that tend to show up in times of "great social, political, and ideological upheaval." If cognitive literary studies is to become truly inter-disciplinary (on which more below), its explanations of difference will have to involve some dialectical interaction between cognitive universals and variations of cultural forms, for example, great social, political, or ideological upheaval. Otherwise, though the reasoning may be convincing, it will still read as if we are being persuaded only that a cognitive affect is present in a literary object, and that is not likely to interest most scholars in the humanities.

With the issue of the kind of significance that can matter, we may turn to the essay of Francis Steen. In setting up his argument Steen writes that "even as the claim that literature has real social effects has attained the status of a truism, the underlying reasons literature works have been left largely unexplored." Of course Steen means that a certain kind of underlying reasons has not been explored, but in any case he will now need to put forth underlying reasons that presumably only become available as a result of insights from some realm of cognitive science. Steen does this by bringing blending theory into play in his consideration of Aphra Behn's Love-Letters between a Nobleman and His Sister (1684).

It seems necessary that any cognitive literary study would be trying to reveal underlying, which is to say unconscious, reasons for some manifest element of the text. This is what will make cognitive literary study interesting. Just as cognitive science reveals the ways the embodied mind works to generate cognition that is part of but also apart from our conscious awareness, so cognitive literary studies would be revealing the ways the embodied mind works as part of but also apart from our conscious awareness with respect to literary phenomena. As we have seen, we will need to show what it can mean that a given cognitive function is at work, and one way to do this is to explain the cognitive function in relation to a historical context, as Steen does in some detail. Care must be taken in doing this, though, because it is easy to make claims about historical change that will be difficult to square with the biology. Steen says of the late seventeenth century that a momentous change was underway "from subject to citizen." But this change was "not simply institutional; it required a new cognitive organization, a new economy of the imagination." If he means that human cognitive architecture as such was undergoing a specifiable historical change, then we have a remarkable claim because it would involve not just a social change but a qualitative biological change in the basic way we apprehend the world. A skeptic may be led to ask: Given Steen's claim, just how fundamental to human being is this concept cognitive? If it is as fundamental as it seems to 
be in the scientific literature, it must change only very seldom and over evolutionarily long periods of time. If the term is not being used in this way, then it ought to be distinguished from its usual cognitive-scientific usages. Otherwise a reader can fail to see how the term cognitive carries a meaningful difference from other possible, nonscientific terms, such as ideology or subjectivity. In fact Steen does not really stay with this large claim, but one should nevertheless be aware of the implications of such a statement.

Again, Steen's argument depends much on blending theory to make its points. He takes off from the basis of a cognitive universal (conceptual blending) and tries to show how this universal can illuminate the nature of certain contradictions in the text. As with other literary-interpretive approaches, focusing on contradictions is rhetorically smart because contradictions automatically need to be explained. He gives us a detailed and convincing reading of the novel, showing how historical contexts can determine the nature of specific literary blends. But as with Turner we have to ask: How necessary to his reading is the cognitive science? Steen understands blending theory and makes a diligent attempt to use it in a comprehensive interpretation, so what I have called the issue of necessity shows up rather clearly. At times Steen stays with the language of blending theory; at times he does not. He writes at one point that the "tension in Behn's dramatization of the story [of the Earl of Berkeley's daughter] relies on the conjunction of a patriarchal perspective on power and a feminist perspective on love. Clearly adapting the father's point of view would have provided a topical and ideologically correct mapping of the [real-world] scandal onto the [real-world] political debate" about the relation of the king to his subjects. "The act of mapping [real-world] politics onto the domain of love opens up a possibility space where the significance of political relations can be transformed" in play, so to speak, without the risks of real-world consequences. This seems true and insightful, but we do not really need cognitive science in order to make these claims. In fact, for long stretches of the essay Steen argues the issues without using words like "mapping" or "domain" and the like. The several-page explication of political and sexual crossovers in the story of Melinda, Silvia, the count, and Philander has little such language and does not need it. The interpretation only needs what it most has: Steen's close reading of the surrounding history, of Behn's biography, and of the novel along with his ability to see analogies between fictional, political, and cultural levels of meaning.

Later on in the explication of this same story we do get what appears to be a return to the science:

In the language of structured connectionism, the rhetoric of the lovers coactivates the domains of love and politics. Each of these have rich sets of logical 
implications. The domain of love calls for associations of the physically erotic, of the natural, of going beyond convention, of passion, compulsion, and irresistible urges. On the other hand, love also involves the dangers of deceit, of guileful seduction bordering on rape with the attendant loss of virginity, honor, reputation, social standing.

Structured connectionism and domain may be necessary terms in the context of psychological theory and practice. But here we might as well say: "In the language of new (or possibly old) historicism, the rhetoric of the lovers is constituted from the discourses of love and politics of the time. Each discourse contributes its own set of elements. The seventeenth-century discourse of love brings in, as do all discursive structures, certain contradictions the covering over of which is a constitutive necessity," and so forth. Or something of the sort. Too often it seems that the vocabulary of cognitive rhetoric is simply being plugged into the interpretation. In fact it begins to seem as if that vocabulary should be either much more prominent or else not present at all. Steen himself almost reveals what I mean when he turns to the work of Dorrit Cohn toward the end of his essay. After quoting some of Cohn's definitions of fictional narrative, Steen says, "Clearly what Cohn describes are cognitive processes as much as formal characteristics of texts." If so, what can we get from the apparatus of cognitive science that we cannot get from Cohn? So far the answer to this question is not clear, but this does not mean the question cannot be answered. Going forward with blending theory will require, I would argue, some further specification of how it operates in the embodied mind, some further empirical understanding of perhaps how it can turn out to be a problem itself as well as a solution to a problem or of how it can conflict with other elements of our cognitive architecture. In the meantime writing such as Steen's shows us where to begin.

\section{Interdisciplinary Necessity and the Historical Study of the Mind/Brain}

Alan Richardson employs cognitive-scientific ideas of the embodied mind to make the case that "the relation between bodies and minds is of more consequence, at least in Persuasion, than critics of Austen have wanted to acknowledge." His argument gives us a clear and well-informed account of debates about the nature of mind in Austen's day. This enables him to show how Austen generated a representation, in both form and content, of an "embodied epistemology" without really knowing that was what she was doing. Further she did so more or less in historical tandem with the emerging "physiological psychologies" of her time.

Once again we have a skilled critic. Novelistic and historical detail come 
together in a solidly convincing argument. Toward the end, however, we read that it "could be objected that this new view of Persuasion, relying as it does primarily on Romantic-era documents, could have been produced without the inspiration of recent neuroscience and cognitive theory." Though some readers might find this self-defeating, I feel it is rhetorically wise. Richardson adequately makes the case that his own reading in cognitive psychology has caused him to see certain textual elements in new ways. And that really is a kind of bottom line for justifying an approach. Once an approach has caused certain textual and historical elements to show up differently, it can often seem that some other approach could have worked as readily. But the key consideration must be whether or not the new perspective actually causes specifiable elements to show up differently than before. I would argue that so far blending theory has failed to do this. Again, the cognitive understanding of blending may have led to a useful method of unpacking metaphor, but it does not really reveal much that would not be revealed by a fine-tuned rhetorical analysis. However, Richardson does in fact notice specific aspects of the text that show up in a new way because of his knowledge of the embodied mind. And yet to his credit, he can sense a certain kind of skeptical reader and so at least tries to anticipate some of the questions I have felt the need to ask. Further, he situates himself at the beginning of the cognitive literary project so that, as with Steen, we can see this kind of work as a phase that will lead to other investigations that will meet this objection.

\section{Interdisciplinarity Looking Forward}

I have saved Lisa Zunshine's essay to the end for reasons that will become apparent. Zunshine sets out to interpret Anna Laetitia Barbauld's Hymns in Prose for Children (1781) "by deploying a conceptual framework made available by recent theoretical breakthroughs in cognitive anthropology," specifically in the area of our evolved, innate ability to distinguish between the categories of "natural kinds and artifacts." She also brings in blending theory. To my mind the use of blending theory seems stronger here, but this is because it is not the focal interdisciplinary concept. Rather, it serves as support for the concept of categorization, which seems to me the strongest interpretive use of a cognitive finding in this volume.

Zunshine is admirably clear in laying out both the general case for cognitive approaches and the specific case for the innate human ability to categorize. Citing work from actual cognitive research by Scott Atran and Frank Keil, she explains that from infancy human beings make a distinction between natural and artificial kinds and that we endow natural kinds with 
an essence we do not ascribe to artificial kinds. She shows how Barbauld represents children as a blend of both natural and artificial kinds. She then shows how the tension embedded in this kind of "ontological ambiguity" gets resolved ideologically. In other words, she brings into play a specific cognitive universal, a specific textual contradiction, and a dialectical relationship between biology and culture. Importantly she also shows how, in more sophisticated texts, the same kind of ambiguity serves to increase rather than overcome tension. As an example she mentions Mary Shelley's Frankenstein (1818), one of the classic representations of a creature that is both an artifact or made creature and a human being, that is, a natural kind. The cognitive explanation here not only provides a convincing new explanation for an old enigma, it also opens the door to other interpretations. We could, with the help of this particular cognitive-anthropological finding, look historically at the wealth of human-but-not-fully-human images in world literature. In other words, we have our category of sameness: a creature that is both an artificial and a natural kind. On this basis, what can we make of the differences in the various versions? If this image always carries, as Zunshine says, a "cognitive challenge," a disorienting element of ontological uncertainty, what makes it more prominent in one time and/or place than in another? What cultural codes are invoked in the particular qualities attributed to a given version of this image?

Finally, Zunshine shows certain contradictions between Barbauld's conscious, folk-psychological understanding of child development-a blank slate ready to be filled-and what she actually writes or implies about the nature of children, which sometimes presumes that children in fact arrive in the world with specific mental predispositions. This compares interestingly to Richardson's essay and shows us two variations on this one approach. Richardson finds a rough parallel between the embodied mind and contemporaneous thinking about psychology; Zunshine finds a contradiction between the two. With these we have an entire new area of investigation opening up. Given the findings of contemporary cognitive studies, we may return to earlier times and look through a new lens at representations, implicit or explicit, of mind-body relations. It will be reasonable to make the working assumption that what we now know of the embodied mind was always present one way or the other in earlier texts. This would be not unlike the way contemporary findings in, say, feminism or psychoanalytic theory enable us to see immanent properties in texts written long before the advent of the theory. How does the embodied mind show up in earlier texts, and what cultural factors are at work to cause it to show up in the precise way that it does? The possibilities are just beginning.

Zunshine, however, does miss an opportunity to distinguish more explic- 
itly between two important categories in this arena of thought. She explains that the tendency to ascribe natural essences to elements of the world "became part of our cognitive makeup" because "it was selected for in thousands of consecutive generations." This, she goes on, "means . . that it remains easy for us - though not at all necessary since nothing about it is 'biologically determined' - to jump to essentialist conclusions when dealing with other human beings." Evidently a cognitive ability is innate but still not rawly determining in a biological way. This is a difference that needs to be made quite clear, at least for a humanities audience, because once again the shadow of social Darwinism looms near. To explain what I think is meant here, we may think of a parallel. It is not just easy but biologically necessary for us in normal conditions to see certain wavelengths of light. Conversely, it is easy but not biologically necessary for us to attribute essences to objects in the world. This brings us to a key quality of the findings of cognitive science. For the most part cognitive science uncovers innate mental structures that have been determined by our formative evolution over thousands of generations. But though these structures are innate and arise from our interactions as animals with the world, the structures are still mental and therefore not rigidly constrained like breathing, walking, metabolism, and so on. Cognitive structures can be violated or vitiated in ways that biological structures cannot. This fact opens the door for nonscientific contributions to a given explanation. Because cognitive structures are not determined in a strictly biological sense, specific cultural practices and ideologies will have a strong part to play in the actual manifestation of a given cognitive universal, and the humanities scholar, one way or another, is already the authority on culture and ideology. Thus we have a stronger possibility of a true interdisciplinarity.

\section{In Conclusion: The Issue of Inter-disciplinarity}

To end my response I want to address a question that regularly mystifies those scholars who find cognitive science so compelling for literary studies: Why has the academic literary establishment not welcomed cognitive studies into the fold? I argue that we encounter a fundamental difficulty with this version of inter-disciplinarity. Knowledge production in whatever academic context involves at least the following three common aspects: it combines a theory with some more (in the sciences) or less (in the humanities) formalized method to drive a practice. Typically we find a dialectical relationship between the theory and practice. Thereby the theory determines the investigative practice, but the practice can, at least in principle, recursively affect the theory. The practice can cause us to feel stronger about the 
validity of the theory, to modify the theory, or possibly to toss it out. Obviously (though the terminology might differ) this is the heart and soul of the empirical sciences, but it operates just as strongly in literary interpretation. Not the theory and practice as such but the epistemological foundations and the nature of the formalized method are what most differentiate humanistic from scientific study. We can find many examples of this in literary and cultural studies. Compare for instance how Michel Foucault's theories of discourse are both employed and changed by someone like Nancy Armstrong (1987) or how Franz Fanon's theories of the psychology of colonialism are both employed and changed by Homi Bhabha (1994).

As far as I can tell, this dialectical relationship between theory and practice cannot be the case with cognitive literary studies because the originating theory cannot, even in principle, be recursively affected by the investigation. We take off from a significantly different theoretical base, namely empirical science of one kind or another. An application of that theory to literature may well change something of our understanding of literature, but it is difficult to see how the interpretive practice can possibly change the theory. If empirical-scientific theory could be changed by what must be considered the nonscientific practice to which we literary scholars put itno controlled experiments, no quantitative data - then little reason would exist to bring it into play in the first place: it would not be substantially different in conceptual kind from the theories that already operate within literary criticism. ${ }^{4}$

For example, as we have seen in Zunshine's essay, cognitive anthropology has established empirically based theories about how humans apprehend natural kinds as opposed to artifacts. But although these theories can of course be disputed, only objections arising either from the same kind of scientific disciplinary practices or from relevant philosophical grounds will have the epistemological weight to affect the theory. Presumably if we set out to interpret a literary text with a theory from cognitive anthropology and begin to find the text itself somehow undermining the theory, we will do mental work to bring the interpretation around to the theory or else give up and start over with some other approach. It is difficult to see how we could legitimately disprove or revise the theory by using it in our usual, nonempirical-scientific interpretive practice. On the other hand, let us take the example of Bhabha's theories about the postcolonial condition. These

4. I would speculate that the obverse interdisciplinarity-say, rhetorical studies of scientific texts-encounters the same problem, and this is part of the reason most scientists dismiss such studies. No dialectic exists between theories of rhetoric on the one hand and science as a kind of truth production on the other; therefore the originating theory cannot be affected by the interpretive practice. 
we could possibly revise or disprove by taking up the actual theories and/or texts he himself used to make his case and showing through the same methods of argument how he has been untrue to either the theories, the texts, or both.

If interdisciplinary tends to mean grounding a practice from one discipline in a theory from another discipline in such a way that theory cannot be affected by practice, then we may have a hard time achieving the truly inter blend that would seem to be the spirit of the term interdisciplinary. The separation of the theory from the investigative practice is just what makes cognitive literary study appealing to some and uninteresting to others. Those who find cognitive approaches appealing will object that, no matter what else, their investigative practice reveals true facts about literature, therefore anyone interested in literature ought to be interested in their findings. But of course, perversely enough, simply being true will not necessarily make a claim or theory interesting. It may also be objected that this kind of interpretation does not actually bear a "practice" relation to cognitive theory in the way I have been using these terms. We take the theory as true and then set out simply to show what new things it can tell us about the world, as opposed to showing by practice the validity of the theory. If so, the problem still remains: we do not really have an inter-discipline.

Failing to have this dialectic does not mean we cannot learn anything new. But it does mean that, unless certain kinds of rhetorical moves are made, cognitive literary study will have a hard time becoming influential. For whatever reasons a truly dialectical relationship between theory, method, and practice seems to provide a basic intellectual appeal to the majority of scholars. The rhetorical moves that will overcome this problem will in general involve what I have tried to make clear above: making the case for the relevance of specific cognitive universals to the study of literary texts, showing how some element of culture and/or psychology relates to a particular version of the given universal, and making the case for the significance of that version in terms of some larger nonscientific concept, such as culture, genre, ideology, politics, or what have you.

This means of course that literary interpretation will remain uncomfortably but intriguingly in between scientific work on one end and artistic work on the other. This seems to me just where interdisciplinary study ought to be.

\section{References}

Armstrong, Nancy

${ }_{1987}$ Desire and Domestic Fiction: A Political History of the Novel (New York: Oxford University Press). 
Bhabha, Homi

1994 The Location of Culture (London and New York: Routledge).

Carroll, Joseph

1995 Evolution and Literary Theory (Columbia: University of Missouri Press).

Damasio, Antonio

1994 Descartes’ Error (New York: G. P. Putnam).

Deacon, Terrence

1997 The Symbolic Species: The Co-Evolution of Language and the Brain (New York: W. W. Norton).

Jackson, Tony E.

2000 "Questioning Interdisciplinarity: Cognitive Science, Evolutionary Psychology, and Literary Criticism," Poetics Today 21: 319-47.

Koestler, Arthur

1964 The Act of Creation (New York: Macmillan).

Storey, Robert

1996 Mimesis and the Human Animal: On the Biogenetic Foundations of Literary Representation

(Evanston, IL: Northwestern University Press).

Turner, Mark

1991 Reading Minds: The Study of English in the Age of Cognitive Science (Princeton, NJ: Princeton University Press).

1996 The Literary Mind (New York: Oxford University Press). 